\title{
Phenomenology of the Normal State of Cu-O High-Temperature Superconductors
}

\author{
C. M. Varma, P. B. Littlewood, and S. Schmitt-Rink \\ AT\&T Bell Laboratories, Murray Hill, New Jersey 07974 \\ E. Abrahams and A. E. Ruckenstein \\ Serin Physics Laboratory, Rutgers University, Piscataway, New Jersey 08855
}

(Received 7 August 1989)

\begin{abstract}
The universal anomalies in the normal state of $\mathrm{Cu}-\mathrm{O}$ high-temperature superconductors follow from a single hypothesis: There exist charge- and spin-density excitations with the absorptive part of the polarizability at low frequencies $\omega$ proportional to $\omega / T$, where $T$ is the temperature, and constant otherwise. The behavior in such a situation may be characterized as that of a marginal Fermi liquid. The consequences of this hypothesis are worked out for a variety of physical properties including superconductivity.

PACS numbers: $74.70 . V y$
\end{abstract}

The normal-state properties of the $\mathrm{Cu}-\mathrm{O}$ superconductors are as perplexing as their high transition temperatures. The electrical resistivity $\rho(T),{ }^{1}$ the thermal conductivity $\kappa(T),{ }^{2}$ the optical conductivity $\sigma(\omega),{ }^{3}$ the Raman scattering intensity $S(\omega),{ }^{4}$ the tunneling conductance as a function of voltage $g(V),{ }^{5}$ the nuclear relaxation rate $T_{1}^{-1}(T),{ }^{6}$ and the Hall coefficient $R_{H}(T)$ (Ref. 7) are all anomalous. Except for the temperature dependence of $R_{H}(T)$, they are also qualitatively the same in all $\mathrm{Cu}-\mathrm{O}$-based high- $T_{c}$ compounds. This collection of properties is unlike those observed in any other metal or expected for a Fermi liquid. At the same time, experiments have revealed that within a resolution of $20 \mathrm{meV}$, these materials have an energy distribution quite consistent with having a Fermi surface. ${ }^{8}$

We shall show that the universal anomalies as well as the appearance of a "Fermi surface" within the stated bounds can be understood from a single hypothesis. The hypothesis is that, over a wide range of momentum $\mathbf{q}$, there exist excitations which make a contribution to both the charge and spin polarizability of the form

$$
\operatorname{Im} \tilde{P}(\mathbf{q}, \omega) \sim\left\{\begin{array}{l}
-N(0)(\omega / T) \text { for }|\omega|<T, \\
-N(0) \operatorname{sgn} \omega \text { for }|\omega|>T,
\end{array}\right.
$$

where $N(0)$ is the unrenormalized one-particle density of states.

Given (1), one finds for the retarded one-particle selfenergy due to exchange of these charge and spin fluctuations

$$
\Sigma(\mathbf{k}, \omega) \sim g^{2} N^{2}(0)\left(\omega \ln \frac{x}{\omega_{c}}-i \frac{\pi}{2} x\right) .
$$

Here, $x=\max (|\omega|, T), \omega_{c}$ is an ultraviolet cutoff, and $g$ is a coupling constant. If Eq. (1) is multiplied by a smooth function of $\mathbf{q}$ which is nonzero over a substantial part of the Brillouin zone, no qualitative change occurs in Eq. (2) or in any of the results we will discuss (see, however, Ref. 9).
Equation (2) is quite different from the one-particle self-energy in a conventional Fermi liquid where, at $T=0, \operatorname{Re} \Sigma \sim \omega$ and $\operatorname{Im} \Sigma \sim-\omega^{2}$ and a quasiparticle representation for the one-particle Green's function is valid:

$$
G(\mathbf{k}, \omega)=\frac{1}{\omega-\epsilon_{\mathbf{k}}-\Sigma(\mathbf{k}, \omega)}=\frac{z_{k}}{\omega-E_{k}+i \Gamma_{k}}+G_{\text {incoh }}
$$

In the present case, we find

$$
z_{k}^{-1}=[1-\partial \operatorname{Re} \Sigma / \partial \omega]_{\omega}=E_{k} \sim \ln \left|\omega_{c} / E_{k}\right|,
$$

near the "Fermi surface" $E_{k}=0$. Thus, according to (3), the quasiparticle weight vanishes logarithmically as

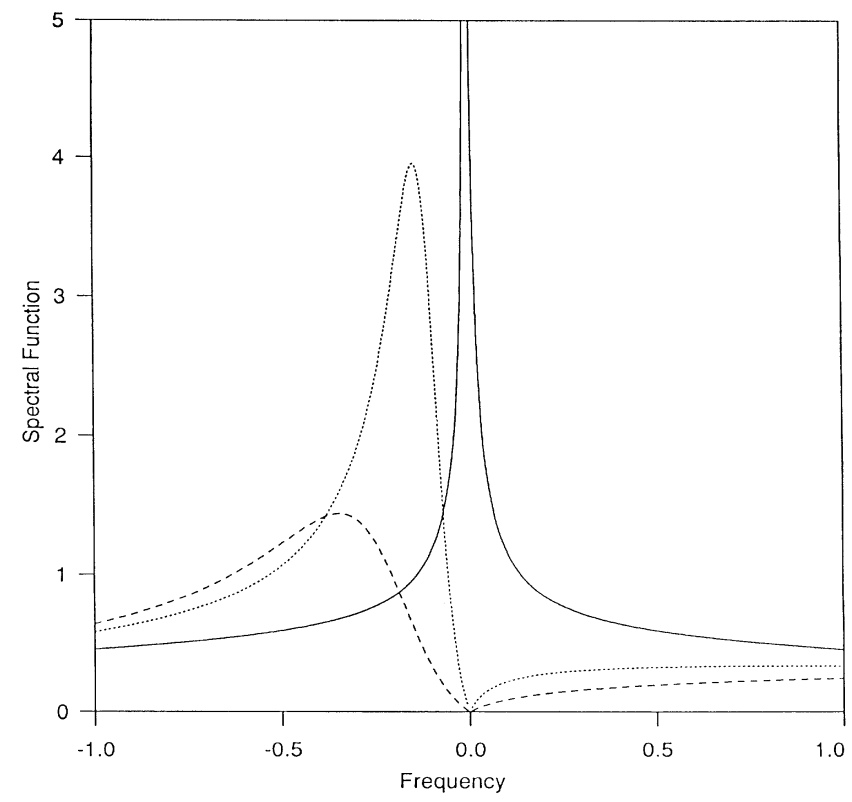

FIG. 1. The spectral function $A(\mathbf{k}, \omega)$ for three values of $\epsilon_{\mathbf{k}}$ : solid line, $\epsilon_{\mathbf{k}}=0$; dotted line, $\epsilon_{\mathbf{k}}=-0.5$; and dashed line, $\epsilon_{\mathbf{k}}$ $=-1.0$ (in units of $\omega_{c}$ ). The coupling constant in Eq. (2) is chosen to be 1 . 
$E_{k} \rightarrow 0$, and $G$ is entirely incoherent. We refer to this as representing a marginal Fermi liquid. Figure 1 shows the single-particle spectral function $A(\mathbf{k}, \omega)$ following from Eq. (2) as a function of $\omega$ for three choices of $\epsilon_{\mathbf{k}}$. The differences of $A(\mathbf{k}, \omega)$ from a normal Fermi liquid are subtle but observable. It is much broader (a normal Fermi liquid, for example, has a $\delta$-function peak when $\epsilon_{\mathrm{k}}=0$ ), and carries substantially more weight in the wings because of the $\omega^{-1}$ tail.

We now discuss the normal-state properties of $\mathrm{Cu}-\mathrm{O}$ superconductors and how they are consistent with the consequences of Eqs. (1)-(3). Only properties that are observed by more than one experimental group and in more than one $\mathrm{Cu}-\mathrm{O}$ compound are considered.

Resistivity $\rho(T)$.- From Eq. (2), a contribution to the resistivity proportional to $T$ is obtained. The normalstate in-plane resistivity of all $\mathrm{Cu}-\mathrm{O}$ superconductors is a constant plus a term linearly proportional to the temperature. $^{1}$ Using $\rho=\left(\omega_{p}^{2} \tau\right)^{-1}$ and rough estimates for the squared plasma frequency $\omega_{p}^{2}$, one finds from the measured slope of $\rho(T)$ that $\tau^{-1}(T) \sim T$, consistent with Eq. (2).

There have been suggestions that the linear temperature dependence of $\rho$ may be the usual behavior from electron-phonon interactions for $T>\Theta_{D} / 4$ where $\Theta_{D}$ is the Debye temperature. Recently, the resistivity of a single crystal of $\mathrm{Bi}_{2} \mathrm{Sr}_{2} \mathrm{CuO}_{6+\delta}$, with $T_{c} \sim 10 \mathrm{~K}$, has been measured. ${ }^{10}$ The temperature dependence of $\rho(T)$ is, to high accuracy, proportional to $T$ from slightly above $T_{c}$ to $600 \mathrm{~K}$. This should eliminate electron-phonon interactions as the source of this behavior.

Tunneling conductance $g(V)$. - The $\mathrm{Cu}-\mathrm{O}$ based superconductors have been found to have a conductance in the normal state given by ${ }^{5}$

$$
g \sim g_{0}+g_{1}|V|,
$$

for $|V|$ between 0 and about $100 \mathrm{meV} . g_{0}$ and $g_{1}$ are very weakly temperature dependent. Quite generally, the current in a tunneling experiment is given by ${ }^{11}$

$$
I=4 \pi e \sum_{\mathbf{k}, \mathbf{k}^{\prime}}\left|M_{\mathbf{k}, \mathbf{k}^{\prime}}\right|^{2} \int_{-\infty}^{\infty} \frac{d \omega_{1}}{2 \pi} A_{1}\left(\mathbf{k}, \omega_{1}\right) \int_{-\infty}^{\infty} \frac{d \omega_{2}}{2 \pi} A_{2}\left(\mathbf{k}^{\prime}, \omega_{2}\right)\left[f\left(\omega_{1}\right)-f\left(\omega_{2}\right)\right] \delta\left(\omega_{1}-\omega_{2}+e V\right),
$$

where $A_{1}$ and $A_{2}$ are the spectral functions for the two materials between which tunneling takes place and $M_{\mathbf{k}, \mathbf{k}^{\prime}}$ is the tunneling matrix element. Suppose 2 is an uninteresting metal with a very slowly varying density of states near the Fermi surface $\left[N_{2}(0)\right]$ and 1 is the metal with interesting many-body effects. Then, with the usual assumption that $M$ is independent of momentum, it is straightforward to show from (5) that

$$
g(V)=4 \pi e^{2}|M|^{2} N_{2}(0) \sum_{\mathbf{k}} A_{1}(\mathbf{k},-e V) .
$$

The tunneling experiments in the normal state are very significant; they state directly that the one-particle density of states, $N(\omega) \sim \Sigma_{\mathbf{k}} A(\mathbf{k}, \omega) \sim N_{0}+N_{1}|\omega|$, is nonanalytic at the chemical potential. It is noteworthy that the photoemission experiment, ${ }^{8(a)}$ which also measures $N(\omega)$ but only for $\omega<0$, has also been fitted with this form with the ratio $N_{0} / N_{1}$ and the slope $N_{1}$ of similar magnitude as in the tunneling.

Since $A(\mathbf{k}, \omega)=-\operatorname{Im} G(\mathbf{k}, \omega) / \pi$, it follows from (6) that a necessary condition for the experimental result (4) is that $\operatorname{Im} \Sigma(\mathbf{k}, \omega) \sim-|\omega|$ as in Eq. (2), in the energy range extending to at least $0.1 \mathrm{eV}$. The sign of $g_{1} \mathrm{de}-$ pends on the $\mathbf{k}$ dependence of $\Sigma$. For example, a positive $g_{1}$ is obtained if an elastic-scattering contribution is added to Eq. (2) with a cutoff smaller than the bandwidth.

Nuclear relaxation rate $T_{1}^{-1}(T)$.- Recent measurements of the in-plane $\mathrm{Cu} T_{1}^{-1}$ in $\mathrm{YBa}_{2} \mathrm{Cu}_{3} \mathrm{O}_{7}$ can be fitted by ${ }^{6}$

$$
T_{1}^{-1}-a T+b,
$$

where the magnitude of $a$ is about a factor of 2 larger than that expected from a Korringa law. The surprise is the temperature-independent term $b,{ }^{9}$ whose value is $b-5 a T_{c}$. If $b$ is interpreted in terms of a characteristic fluctuation time $\tau_{m}$ via $b \equiv 2 \pi\left|H_{\mathrm{hf}}\right|^{2} \tau_{m}$, where the hyperfine coupling $\left|\boldsymbol{H}_{\mathrm{hf}}\right|$ has been independently measured, then $\tau_{m}$ turns out to be $\sim 1 \mathrm{eV}^{-1}$.

Quite generally,

$$
T_{1}^{-1} \sim-\lim _{\omega \rightarrow 0} \frac{T}{\omega} \sum_{\mathbf{q}} \operatorname{Im} \chi_{m}^{+-}(\mathbf{q}, \omega),
$$

where $\chi_{m}^{+-}$is the transverse magnetic susceptibility. We have two important contributions to $\chi_{m}^{+-}: \tilde{P}$ from Eq. (1), and the contribution from particle-hole pairs renormalized according to Eq. (2). In spite of the nonFermi-liquid character of the single-particle propagator, Eqs. (2) and (3), the latter yields the term proportional to $T$ (up to logarithmic corrections) in Eq. (7), just as for ordinary metals. However, the contribution $\tilde{\boldsymbol{P}}$ yields the constant term in Eq. (7), with $\tau_{m} \sim N(0)$. Below, we will encounter the necessity of including both these contributions again, when we consider the optical conductivity. We have used $\tilde{P}$, Eq. (1), to denote the leading frequency contribution of renormalized particle-hole excitations with the complete vertex; the other term is the leading frequency contribution of renormalized particlehole excitations without any vertex correction. Such a decomposition is always possible.

Specific heat $C_{v}(T)$ and thermal conductivity $\kappa(T)$.- The electronic contributions to $C_{v}$ in the normal state cannot be extracted from the data with high accuracy because the contribution of phonons is very large above the high $T_{c}$ of these materials. $\kappa(T)$ in the normal state is observed to be nearly temperature independent. ${ }^{2}$ Since the conductivity is proportional to $T^{-1}$, the Wiedemann-Franz law $\kappa(T) / \sigma(T) T=$ const is obeyed if 
we assume that the observed $\kappa(T) \approx$ const is electronic. The Wiedemann-Franz law holds if the scattering processes for $\sigma(T)$ and $\kappa(T)$ are the same and the specific heat varies linearly with temperature. If the self-energy of Eq. (2) is used to calculate the entropy, logarithmic corrections to the linear-in- $T$ dependence are found. The observed $K / T \sigma$ ratio allows such a weak correction.

Raman scattering. - The most notable feature of the Raman scattering intensity in the normal phase of superconducting samples is a large featureless, nearly frequency- and temperature-independent background extending to about $\frac{1}{2} \mathrm{eV} .{ }^{4}$ The Raman intensity is

$$
\begin{aligned}
I(\omega) & \sim-[1+n(\omega)] \operatorname{Im} \epsilon^{-1}(0, \omega), \\
& \sim\left\{\begin{array}{l}
-(T / \omega) \operatorname{Im} P(0, \omega) \text { for } \omega<T, \\
-\operatorname{Im} P(0, \omega) \text { for } \omega>T .
\end{array}\right.
\end{aligned}
$$

Inserting Eq. (1), a constant background is immediately obtained. This has consequences for superconductive pairing which we discuss below. Other contributions to $P$ give the usual peak near the plasma frequency.

Optical conductivity $\sigma(\omega)$.- From (1) and (2) (as in the discussion for $\left.T_{1}^{-1}\right), \sigma(\omega)$ is the sum of two terms,

$$
\sigma(\omega)=\sigma_{1}(\omega)+\sigma_{2}(\omega) .
$$

$\sigma_{i}(\omega)$ is the Drude contribution

$$
\sigma_{1}(\omega) \sim \operatorname{Im} \omega_{\mathrm{pl}}^{2} /[\omega+i \Sigma(\omega)],
$$

$\sigma_{2}(\omega)$ is the direct absorption due to (1) itself

$$
\sigma_{2}(\omega) \sim-\omega \operatorname{Im} \tilde{P}(0, \omega) .
$$

The integrated oscillator strength, $\omega_{\mathrm{pl}}^{2}$ in (11), plus that in (12), must add to the total band structure value $\omega_{p}^{2}$. In Fig. 2, we compare the calculated $\sigma(\omega)$ using (10), with $\omega_{\mathrm{pl}} / \omega_{p}$ and $\omega_{c}$ as adjustable parameters, with experiment. $^{3}$ There is a peak around zero frequency of width $\sim T ; \sigma(\omega)$ then increases slowly on a scale of $T$ and declines as $\omega^{-1}$ on a scale of $\omega_{c}$.

Photoemission-As already mentioned, the deductions from (1) and (2) are consistent with the measurements of $N(\omega)$ in high-resolution photoemission experiments. ${ }^{8}$ The differences of the spectral function $A(\mathbf{k}, \omega)$ following from Eq. (2), shown in Fig. 1, from that for a normal Fermi liquid are measureable in an angleresolved photoemission experiment.

Superconductive pairing. - The particle-particle scattering amplitude, which is the pairing kernel in a theory of superconductivity, is given approximately by $V(q)$ $\times \operatorname{Re} \epsilon^{-1}(q, \omega)$, where $V(q)$ is the bare interaction. As we discussed, the measured $\operatorname{Im} \epsilon^{-1}(0, \omega) \sim \operatorname{Im} P(0, \omega)$ in Raman scattering has precisely the form of Eq. (1) up to a (soft) cutoff, say $\omega_{c}$ of the order of few tenths of an eV. The agreement with experiment which we have found supports our initial hypothesis of the $q$ independence of $\tilde{P}$ over a substantial range. The sign and behavior of $\operatorname{Im} \epsilon^{-1}$ which follows from these considerations

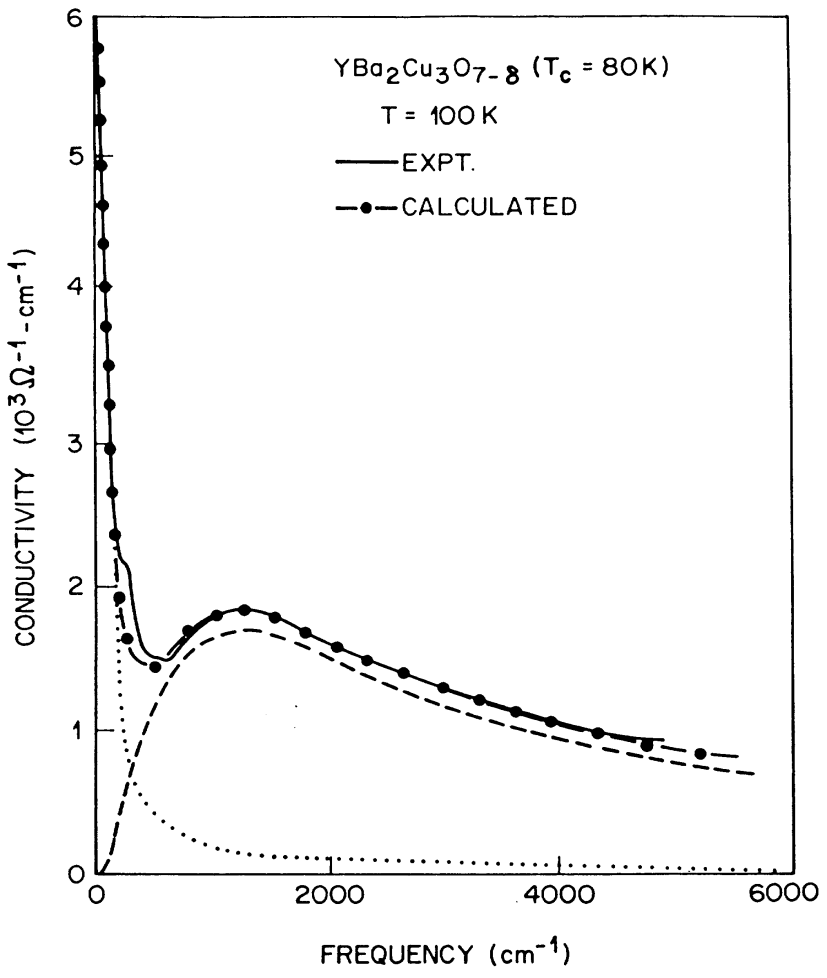

FIG. 2. The frequency-dependent conductivity from Ref. 3(b) is shown together with the theoretical fit based on Eq. (10). For this fit Eq. (1) is parametrized as $\operatorname{Im} \tilde{P}(q, \omega)$ $\sim-\tanh (\beta \omega / 2)\left(1+\omega^{2} / \omega_{c}^{2}\right)^{-1}$ with $\omega_{c} \approx 1200 \mathrm{~cm}^{-1}$ and $\omega_{\mathrm{pl}}^{2} /$ $\omega_{p}^{2}=1 / 5$. The dotted curve gives the contribution of $\sigma_{1}$; the dashed curve that of $\sigma_{2}$. The dash-dotted curve is the total.

leads, by the Kramers-Kronig relation, to a negative contribution to $\operatorname{Re}\left[\epsilon^{-1}(q, \omega)-1\right]$ over a sizable region of $q$ and $\omega<\omega_{c}$. It thus appears likely that the excitations represented by Eq. (1) are responsible both for $s$-wave pairing consistent with observations, ${ }^{12}$ and for the anomalous normal-state properties. Contributions from other processes, in general, ensure that $\operatorname{Re} \epsilon^{-1}(q, 0)>0$ as required for stability. These contributions presumably extend on a scale of the bandwidth $\gg \omega_{c}$, so that the attractive contribution for $\omega<\omega_{c}$ can win out for pairing.

Conclusions. - A single hypothesis, Eq. (1), unifies the universal features of the normal state of $\mathrm{Cu}-\mathrm{O}$ based superconductors. This hypothesis characterizes these materials in the normal state as marginal Fermi liquids, and leads, as well, to an attractive particle-particle interaction for superconductive pairing.

The success of the phenomenology based on Eq. (1) puts at least two constraints on microscopic theories. Equation (1) demands that the charge and spin susceptibility have the same functional form; the fit to experiments, say $T_{1}^{-1}$ and $\sigma(\omega)$, necessitates that they are even similar in magnitude. It seems to be inescapable that charge and spin degrees of freedom have similar en- 
ergy scales in the metallic phase of $\mathrm{Cu}-\mathrm{O}$ based materials.

The other important conclusion follows from Eqs. (1) and (2). Such a behavior can only be obtained if perturbation theory, starting from a noninteracting Fermi gas, breaks down well above the eventual superconducting transition temperature. A singularity with a large scale has indeed been found in particle-particle scattering in 2D Fermi gases with attractive interactions. ${ }^{13}$ More work is needed to see if it leads to Eq. (1).

We wish to thank numerous experimentalist colleagues for many patient and helpful discussions. E.A. wishes to acknowledge stimulating discussions with $\mathbf{P}$. W. Anderson. E.A.'s research was supported in part by NSF Grant No. DMR 85-20190-01.

${ }^{1}$ See, for example, M. Gurvitch and A. T. Fiory, Phys. Rev. Lett. 59, 1337 (1987).

${ }^{2}$ See, for example, F. Steglich, in Materials and Mechanisms of Superconductivity, edited by J. Müller and J. L. Olsen (North Holland, Amsterdam, 1988).

${ }^{3}$ (a) For a recent review, see T. Timusk and D. B. Tanner, in The Physical Properties of High Temperature Superconductors, edited by D. M. Ginsberg (World Scientific, Singapore, 1987); (b) G. A. Thomas et al., Phys. Rev. Lett. 61, 1313 (1988).
${ }^{4}$ For a review, see S. Sugai, in Mechanisms of High Temperature Superconductivity, edited by H. Kamimura and A. Oshiyama (Springer-Verlag, Heidelberg, 1989).

${ }^{5}$ For a review, see M. Lee, A. Kapitulnik, and M. R. Beasley, in Ref. 4. For more recent work, see, for example, M. Gurvitch et al., Phys. Rev. Lett. 63, 1008 (1989).

${ }^{6}$ See, for example, articles by R. E. Walstedt and W. Warren, in Ref. 4; Y. Kitaoka et al., in Ref. 4; and H. Yasuoka, in Ref. 4.

${ }^{7}$ See, for example, A. Zettl et al., in Ref. 4.

${ }^{8}$ (a) J.-M. Imer et al., Phys. Rev. Lett. 62, 336 (1989); (b) C. G. Olson et al. (to be published).

${ }^{9}$ In contrast to $T_{1}^{-1}$ for $\mathrm{Cu}, T_{1}^{-1}$ for $\mathrm{O}$ is consistent with a Korringa rate or at best with a $b$ term which, appropriately normalized, is only $\frac{1}{6}$ of that for $\mathrm{Cu}$. [M. Takigawa (private communication); R. Walstedt (private communication)]. This behavior can only be obtained from a appropriate $q$ dependence of $\tilde{\boldsymbol{P}}$. As mentioned earlier, if a smooth function of $\mathbf{q}$ multiplies Eq. (1), none of the results discussed here are altered. Discussion of the differences between $T_{1}^{-1}$ for $\mathrm{O}$ and $\mathrm{Cu}$ requires a detailed microscopic theory for $\tilde{P}$ for $\mathrm{Cu}-\mathrm{O}$ metals.

${ }^{10} \mathrm{~S}$. Martin et al. (private communication).

${ }^{11}$ See, for example, E. Wolf, Principles of Electron Tunneling Spectroscopy (Oxford Univ. Press, New York, 1985).

${ }^{12}$ D. R. Harshman et al., Phys. Rev. B 39, 851 (1989); L. Krusin-Elbaum et al., Phys. Rev. Lett. 62, 217 (1989).

${ }^{13}$ S. Schmitt-Rink, C. M. Varma, and A. E. Ruckenstein, Phys. Rev. Lett. 63, 445 (1989). 